\title{
Avaliação da perda de massa de farinhas precursoras de clínquer CSAB compostas com lodo de anodização do alumínio
}

\section{Mass loss evaluation of CSAB clinker raw materials composed with aluminum anodization sludge.}

Eugênio Bastos da Costa $^{1}$, Maurício Mancio ${ }^{2}$, Antonio S. Takimi ${ }^{3}$, Ana Paula Kirchheim ${ }^{4}$

\author{
${ }^{1,4}$ Programa de Pós-Graduação em Engenharia Civil - Universidade Federal do Rio Grande do Sul - NORIE/UFRGS CP: \\ 90035-190, Porto Alegre, RS \\ ebc.eng@hotmail.com \\ ${ }^{2}$ Programa de Pós-Graduação em Engenharia Civil - PPGEC - Universidade do Vale do Rio dos Sinos - UNISINOS - \\ CP: 93022-000, São Leopoldo - RS \\ ${ }^{3}$ Programa de Pós-Graduação em Ciência e Engenharia de Materiais - PPGCEM/UFPel CP: 96010-000, Pelotas, RS \\ e-mail: mancio@unisinos.br; antonio.takimi@gmail.com; anapaula.k@ufrgs.br
}

\section{RESUMO}

O desenvolvimento científico do cimento Portland é bastante difundido no Brasil e no mundo. Entretanto, o cimento sulfoaluminato de cálcio belítico (CSAB), embora seja considerado de menor impacto ambiental, ainda têm como base estudos em fase relativamente precoce em relação aos já desenvolvidos para o cimento Portland mundialmente e com menor expressão ainda no âmbito nacional. Na produção do clínquer CSAB, produto base para a produção deste cimento, uma questão relevante está relacionada ao conteúdo de enxofre presente na farinha precursora, que é maior que no clínquer Portland. Materiais percursores a base de enxofre são necessários para formação de fases como a ye'elimita e anidrita, sendo que em quantidades inadequadas podem ser volatilizados subprodutos a base de enxofre que geram transtornos operacionais e ambientais. $\mathrm{O}$ excesso de enxofre na matéria-prima do cimento Portland pode ocasionar a elevação da emissão de $\mathrm{SO}_{2} \operatorname{com}$ os gases de saída, abafando a suspensão do pré-aquecedor, além de provocar a formação de anéis de colagem no forno, escapando para atmosfera o $\mathrm{SO}_{2}$, o que pode contribuir para formação de chuvas ácidas. Além disso, a viabilização da produção do cimento CSAB depende da disponibilidade de matérias-primas a baixo custo, sendo o aproveitamento de resíduos industriais uma alternativa. Dentre os resíduos com potencial de uso em farinhas precursoras de clínquer CSAB destaca-se o lodo de anodização de alumínio, uma rica fonte de alumínio, elemento importante para formação desses cimentos. Assim, é importante avaliar o comportamento frente à perda de massa de farinhas precursoras de clínquer CSAB sintetizadas a partir de lodo de anodização do alumínio para verificar a influência do resíduo na decomposição de fases sulfatadas acima da temperatura de formação da ye' elimita $\left(1250^{\circ} \mathrm{C}\right)$. Foram compostas farinhas com reagentes químicos puros, com resíduo e com materiais convencionais. As matérias-primas foram proporcionadas por equações potenciais de formação de fases sintéticas a partir da composição de óxidos da farinha. Para verificação da perda de massa, foram realizadas análises termogravimétricas no intervalo de temperatura compreendido entre 30 e $1450^{\circ} \mathrm{C}$. $\mathrm{O}$ estudo comprovou a potencialidade no uso de resíduos do processamento de alumínio para produção de cimentos CSAB e se verificou que há um limite de temperatura próximo aos $1250^{\circ} \mathrm{C}$ para evitar decomposição de fases sulfatadas e a consequente emissão de $\mathrm{SO}_{2}$.

Palavras-chave: cimento sulfoaluminato de cálcio; cimento belítico; emissão de $\mathrm{CO}_{2} ; \mathrm{SO}_{2}$; coprocessamento.

\section{ABSTRACT}

Portland cement science is well established in Brazil and in the world; however, the development of calciumsulfoaluminate belite (CSAB) cements, considered a low-environmental impact material, is still in its initial stages relative to cement Portland abroad and there are even fewer studies nationally. One concern regarding the production of CSAB cements is the high amount of sulfur in the raw materials. Excess sulfur in the feed- 
stock of Portland cement causes the increase of $\mathrm{SO}_{2}$ emissions, a dampening the efficiency of the pre-heater in the clinker factory, in addition to causing the formation of bonding rings inside the kiln. The viability of the production of CSAB cement depends on the availability of raw materials at low cost, preferably with the use of an alternative industrial waste. Thus, the aim of this work is to analyze the mass loss of lab-made CSAB mixtures up to a temperature of $1450^{\circ} \mathrm{C}$, in order to verify the decomposition of sulfated compounds and to evaluate the possibility of replacing bauxite by an alternative source of $\mathrm{Al}_{2} \mathrm{O}_{3}$, namely by aluminum anodizing sludge (AAS) residue, which presents itself as an interesting raw-material for the production of CSAB clinker due to its high content of alumina. Mixtures made with pure chemicals, AAS residue and conventional materials were produced. Raw materials were proportioned based on equations formation of compounds synthetic from the mixture oxides composition. To check the mass loss, thermogravimetric analyzes were performed. It was concluded that the maximum temperature for CSAB clinker production should be close to $1250^{\circ} \mathrm{C}$ to avoid decomposition of sulfur-containing phases and their related $\mathrm{SO}_{2}$ emissions.

Keywords: sulfoaluminate cement; belite cement; $\mathrm{CO}_{2}$ emissions; co-processing.

\section{INTRODUÇÃO}

Segundo ROCHA et al. [1], o processo de fabricação do cimento Portland é, essencialmente, a calcinação e a fusão de um material constituído aproximadamente de $94 \%$ de calcário, $4 \%$ de argilas e $2 \%$ de óxidos de ferro e alumínio em um forno rotativo operando em temperaturas de $1450^{\circ} \mathrm{C}$ para os sólidos, em que a temperatura de chama oscila em torno de $2000^{\circ} \mathrm{C}$. Para facilitar a formação das fases desejadas no clínquer de cimento Portland, ou alternativos que sejam produzidos no sistema forno, é necessário que a mistura de matérias-primas esteja finamente moída e bem homogeneizada antes do tratamento térmico. A partir da análise química das pilhas de materiais estocados, as suas proporções individuais são determinadas pela composição desejada no produto final; as matérias-primas proporcionadas são geralmente moídas em moinho de bolas ou de rolo até partículas geralmente menores que $75 \mu \mathrm{m}$. A mistura assim obtida é também denominada farinha [2]

O processo de clinquerização representa uma demanda energética de $24,2 \mathrm{kWh} / \mathrm{t}$, correspondente a $22 \%$ da energia elétrica consumida durante o processo produtivo do cimento [ $\underline{3}-4$ ]. No Brasil, a partir de dados da USGS (United States Geological Survey ou Pesquisa Geológica dos Estados Unidos) e EIA (US Energy Information Administration ou Administração de Informações sobre Energia dos Estados Unidos), calcula-se que a fabricação de cimento é responsável por cerca de $9,3 \%$ da geração nacional de $\mathrm{CO}_{2}$ a partir da queima de combustíveis fósseis [믐․ Se comparadas às emissões de $\mathrm{CO}_{2}$ causadas pelo consumo de gasolina e álcool combustível no país, verifica-se que as emissões devido à fabricação de cimento correspondem a aproximadamente $55 \%$ deste total $[\underline{7}, \underline{8}, \underline{9}]$.

A emissão química de $\mathrm{CO}_{2}$ no processamento de cimentos está associada à decomposição da calcita $\left(\mathrm{CaCO}_{3}\right)$ presente no calcário em torno dos $800^{\circ} \mathrm{C}$, gerando como subproduto o $\mathrm{CO}_{2}$. A partir da estequiometria dessa reação pode-se estimar que a cada tonelada de calcita calcinada, $440 \mathrm{~kg}$ de $\mathrm{CO}_{2}$ são liberados para a atmosfera. Em comparação com o Portland, a farinha precursora do clínquer Sulfoaluminto de cálcio belítico (CSAB), devido sua química diferenciada, apresenta uma reduzida emissão de $\mathrm{CO}_{2}$, além de adquirir suas características finais desejadas a uma temperatura $200^{\circ} \mathrm{C}$ inferior ao do cimento Portland, que é tipicamente em torno dos $1450^{\circ} \mathrm{C}$. Essa redução está associada à composição de óxidos e de fases do CSAB [17]. A Tabela 1 apresenta uma comparação das variações composicionais e temperatura de formação das fases dos cimentos Portland e CSAB.

Tabela 1: Compostos principais dos cimentos Portland e CSAB, variação da composição média destas fases, conteúdo de $\mathrm{CaO}$ em fases de cimentos e temperaturas de sintetização.

\begin{tabular}{l|l|l|l|l}
\hline $\begin{array}{l}\text { COMPOSTO/ } \\
\text { COMPOSIÇÃo EM ÓXIDOS }\end{array}$ & $\begin{array}{l}\text { ABREVIAÇÃO/ } \\
\text { \%ÓXIDO DE CÁLCIO }\end{array}$ & $\begin{array}{l}\text { TEMPERATURA DE } \\
\text { SINTETIZAÇÃO }\end{array}$ & $\begin{array}{l}\text { PORTLAND } \\
\text { (TAYLOR [15], } \\
\text { 1992) }\end{array}$ & $\begin{array}{l}\text { CSAB } \\
\text { (ODLER [16], } \\
\text { 2000) }\end{array}$ \\
\hline $\begin{array}{l}\text { Silicato tricálcico/ } \\
3 \mathrm{CaO} . \mathrm{SiO}_{2}\end{array}$ & $\begin{array}{l}\mathrm{C}_{3} \mathrm{~S}-\text { alita/ } \\
73,7 \%\end{array}$ & $\approx 1450^{\circ} \mathrm{C}$ & $50-70 \%$ & $0 \%$ \\
\hline $\begin{array}{l}\text { Silicato dicálcico/ } \\
2 \mathrm{CaO} . \mathrm{SiO}_{2}\end{array}$ & $\begin{array}{l}\mathrm{C}_{2} \mathrm{~S}-\text { belita/ } \\
65,1 \%\end{array}$ & $1000-1200^{\circ} \mathrm{C}$ & $15-30 \% ;$ & $10-60 \%$ \\
\hline Aluminato tricálcico/ & $\mathrm{C}_{3} \mathrm{~A}-$ aluminato/ & $>1200^{\circ} \mathrm{C}$ & $5-10 \% ;$ & $0 \%$ \\
\hline
\end{tabular}




\begin{tabular}{l|l|l|l|l}
\hline $3 \mathrm{CaO} \cdot \mathrm{Al}_{2} \mathrm{O}_{3}$ & $62,2 \%$ & & & \\
\hline $\begin{array}{l}\text { Ferro aluminato tetracálcico/ } \\
4 \mathrm{CaO} \cdot \mathrm{Al}_{2} \mathrm{O}_{3} \cdot \mathrm{Fe}_{2} \mathrm{O}_{3}\end{array}$ & $\begin{array}{l}\mathrm{C}_{4} \mathrm{AF}-\text { ferrita/ } \\
46,2 \%\end{array}$ & $>1000^{\circ} \mathrm{C}$ & $5-15 \%$ & $0-40$ \\
\hline $\begin{array}{l}\text { Sulfato trialuminato tetracálcico/ } \\
4 \mathrm{CaO} .3 \mathrm{Al}_{2} \mathrm{O}_{3} \cdot \mathrm{CaSO}_{4}\end{array}$ & $\begin{array}{l}\mathrm{C}_{4} \mathrm{~A}_{3} \$-\text { ye'elimita/ } \\
36,7 \%\end{array}$ & $1000-1250^{\circ} \mathrm{C}$ & $0 \%$ & $10-55 \%$ \\
\hline
\end{tabular}

O cimento CSAB ainda está em fase de testes, não tendo sido ainda utilizado em obras. No entanto vários grupos de pesquisa atuam nesta linha, como CHEN [10], JEWELL et al. [11], MARTÍN-SEDEÑO et al. [12], SENFF et al. [13], ÁLVAREZ-PINAZO et al. [14]. A nível industrial a empresa Lafarge vem divulgando o desenvolvimento de uma nova linha de cimentos CSAB, a qual patentearam sob o nome de AETHER $®$, no entanto as pesquisas de desenvolvimento do produto ainda estão sendo realizadas e a primeira produção industrial do produto foi divulgada em 2013. A empresa justifica que o projeto possibilitará a redução da emissão de $33 \%$ de $\mathrm{CO}_{2}$ por tonelada de cimento até o ano de 2020.

Segundo o estudo de COSTA [17], que comparou a emissão química de $\mathrm{CO}_{2}$ entre cimentos CSAB e Portland considerando a descarbonação da farinha precursora, o primeiro teria uma emissão máxima da ordem de $327 \mathrm{~kg} \mathrm{CO}_{2} /$ ton de cimento, a depender do teor de óxido de cálcio e trióxido de enxofre do produto, já o Portland emite $501 \mathrm{~kg} \mathrm{CO}_{2} /$ ton de clínquer, essa emissão pode ser minimizada pelo emprego de adição de materiais pozolânica, carbonáticos ou minerais na produção de cimento Portland, reduzindo o fator clínquer. Os cimentos sulfoaluminatos de cálcio belíticos contêm as fases belia $\left(\mathrm{C}_{2} \mathrm{~S}\right)$ e ye'elimita $\left(\mathrm{C}_{4} \mathrm{~A}_{3} \$\right)$ como seus principais constituintes [16]. Segundo o autor, eles não contêm alita $\left(\mathrm{C}_{3} \mathrm{~S}\right)$ ou aluminato tricálcico $\left(\mathrm{C}_{3} \mathrm{~A}\right)$, mas podem possuir quantidades variadas de ferroaluminato tetracálcico $\left(\mathrm{C}_{4} \mathrm{AF}\right)$, bem como sulfato de cálcio em quantidades maiores que o normal encontrado em cimentos Portland. Algumas fases menores também podem estar presentes, tais como: $\mathrm{CA}, \mathrm{C}_{12} \mathrm{~A}_{7}, \mathrm{M}$ (periclásio), $\mathrm{C}_{2} \mathrm{AS}$ (gehlenite), e $\mathrm{C}_{5} \mathrm{~S}_{2} \$$ (sulfospurite ou ternesita) [18]. Acima da temperatura de $1200^{\circ} \mathrm{C}$ pode ocorrer a decomposição do $\mathrm{C}_{4} \mathrm{~A}_{3} \$$, reações com a cal livre, caso presente, para formar $\mathrm{C}_{3} \mathrm{~A}$ e $\mathrm{CaSO}_{4}[16]$.

Um dos pontos negativos da produção deste cimento alternativo, é a necessidade de elevada quantidade de $\mathrm{Al}_{2} \mathrm{O}_{3}$ para a produção da farinha precursora do clínquer CSAB. Segundo CHEN [10], depósitos de bauxita geralmente são reservados para uso na produção de alumínio e alumina, o que onera a produção destes cimentos, principalmente pela indisponibilidade a baixo custo de matérias-primas ricas em $\mathrm{Al}_{2} \mathrm{O}_{3}$. Apesar de apresentar vantagens ambientais quanto à emissão de $\mathrm{CO}_{2}$, um grande entrave se apresenta para a produção em larga escala, devido à necessidade de materiais alternativos a bauxita. Além disso, o conteúdo de sulfato na farinha para sintetização do $\mathrm{C}_{4} \mathrm{~A}_{3} \$$ é outro fator preocupante para a sua produção, visto que o excesso de enxofre na matéria-prima de cimentos Portland pode ocasionar a elevação da emissão de $\mathrm{SO}_{2}$ com os gases de saída, abafando a suspensão do pré-aquecedor, além de provocar a formação de anéis de colagem no forno [19].

Segundo CHEN [10], por outro lado é desejável encontrar matérias-primas alternativas para a produção do cimento CSAB para manter os custos competitivos e reduzir ainda mais o impacto ambiental através da utilização de resíduos industriais.

Desta forma, considerando os benefícios que podem ser atingidos com o uso do cimento CSAB, e também as barreiras ainda existentes e os problemas que podem ser ocasionados pelo seu mau uso, considera-se importante um estudo aprofundado nesta direção. Assim, neste trabalho são analisadas amostras de farinhas CSAB produzidas em escala laboratorial quanto à perda de massa até a temperatura de $1450^{\circ} \mathrm{C}$ para verificação da decomposição de compostos sulfatados. Os clínqueres analisados foram produzidos com e sem bauxita, tendo sido esta substituída total e parcialmente por lodo de anodização do alumínio (LAA), resíduo que se apresenta como uma alternativa interessante para a produção do clínquer CSAB, pois possui alto teor de $\mathrm{Al}_{2} \mathrm{O}_{3}$ quando seco ou calcinado.

\section{MATERIAIS E MÉTODOS}

A composição química das matérias-primas foi determinada por fluorescência de raios X (FRX), realizada com aparelho marca Shimadzu, modelo XRF1800 do Laboratório de Materiais Cerâmicos da Universidade Federal do Rio Grande do Sul (LACER/UFRGS).

Foram utilizadas equações de BOGUE [20] adaptadas por MAJLING e ROY [21] para o proporcionamento de materiais para a composição das farinhas precursoras de clínqueres CSAB. As equações de BOGUE [19] adaptadas são utilizadas para a quantificação das fases do clínquer através da composição de óxi- 
dos da farinha, ver equações de 1 a 5 .

(1)

(2)

$$
\% \mathrm{C}_{4} \mathrm{AF} \quad=\quad 3,043\left(\% \mathrm{Fe}_{2} \mathrm{O}_{3}\right)
$$

$$
\begin{array}{lllll}
\% \mathrm{C}_{4} \mathrm{~A}_{3} \$ & = & 1,995\left(\% \mathrm{Al}_{2} \mathrm{O}_{3}\right) & - & 1,273\left(\% \mathrm{Fe}_{2} \mathrm{O}_{3}\right)
\end{array}
$$

$$
\begin{array}{lll}
\% \mathrm{C}_{2} \mathrm{~S} & = & 2,867\left(\% \mathrm{SiO}_{2}\right)
\end{array}
$$

$$
\% \mathrm{C} \$ \quad=\quad-700\left(\% \mathrm{SO}_{3}\right)+0,445\left(\% \mathrm{Al}_{2} \mathrm{O}_{3}\right)+\quad+\quad 0,284\left(\% \mathrm{Fe}_{2} \mathrm{O}_{3}\right)
$$$$
\% \mathrm{C}=1,000(\% \mathrm{CaO})-1,867\left(\% \mathrm{SiO}_{2}\right)-1,054\left(\% \mathrm{Fe}_{2} \mathrm{O}_{3}\right)-0,550\left(\% \mathrm{Al}_{2} \mathrm{O}_{3}\right)-0,700\left(\% \mathrm{SO}_{3}\right)
$$

Chen [10] utilizou equações complementares às de BOGUE [20] para estimar o teor de periclásio e de outras impurezas, ver equações 6 e 7 :

$\% \mathrm{MgO}=1,00(\% \mathrm{MgO})$

$\%$ Outras Impurezas = 1,00 (\%Outras Impurezas)

Inicialmente foi realizado o quarteamento dos resíduos, preparação e pesagem das matérias primas e mistura das farinhas como é indicado na Figura 1a, 1b, 1c e 1d abaixo.
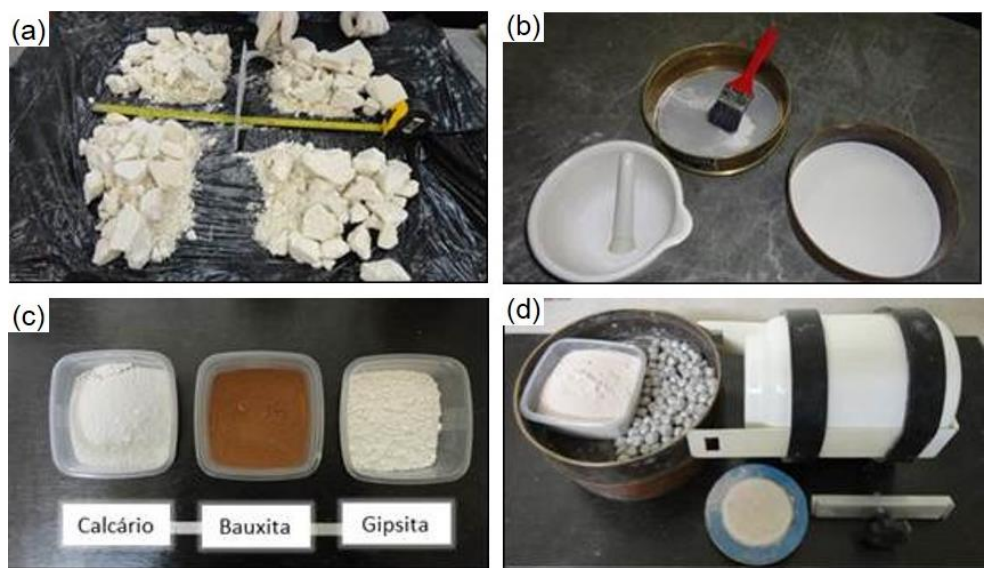

Figura 1: Quarteamento do LAA (a). Amostra do LAA seco, peneirado e usado para substituir a bauxita (b), matérias primas convencionais (c), e Farinha após o processo de mistura das matérias primas (d).

A avaliação da perda de massa das farinhas precursoras dos clínqueres propostos foi realizada a partir de análises termogravimetricas realizadas no LACER/UFRGS em um equipamento Mettler Toledo TGA/TSO SDTA $851 \mathrm{e} \mathrm{com} \mathrm{aquecimento} \mathrm{a} \mathrm{partir} \mathrm{da} \mathrm{temperatura} \mathrm{ambiente} \mathrm{até} 1450^{\circ} \mathrm{C}$, operando a uma taxa de aquecimento de $10^{\circ} \mathrm{C} / \mathrm{min}$, em atmosfera de nitrogênio. Para avaliação da morfologia e da composição elementar das fases dos clínqueres experimentais foram realizadas análises MEV-EDS utilizando um equipamento Hitachi TM 3000, do Laboratório de Design e Seleção de Materiais (LdSM) da UFRGS, com magnificação de até $30.000 \mathrm{X}$ e aceleração de voltagem variável de $5 \mathrm{kV}$ e $15 \mathrm{kV}$ sob atmosfera e taxa de aquecimento de $10^{\circ} \mathrm{C} / \mathrm{min}$.

\section{RESULTADOS E DISCUSSÕES}

\subsection{Composição química, de fases sintéticas e proporcionamento de materiais}

A Tabela 2 mostra a composição de óxidos de todas as matérias-primas utilizadas. Observa-se que o LAA seco tem $73 \%$ de $\mathrm{Al}_{2} \mathrm{O}_{3}$ e $20 \%$ de $\mathrm{SO}_{3}$, deste modo esse resíduo tanto pode suprir parcela da demanda de sulfato quanto total de alumina na composição de farinhas CSAB. 
Tabela 2: Composição de óxidos das matérias-primas utilizadas na composição da farinha precursora.

\begin{tabular}{l|l|l|l|l|l|l|l}
\hline MATÉRIAS-PRIMAS & $\mathrm{SiO}_{2}(\%)$ & $\mathrm{Al}_{2} \mathrm{O}_{3}(\%)$ & $\mathrm{Fe}_{2} \mathrm{O}_{3}(\%)$ & $\mathbf{S O}_{3}(\%)$ & $\mathrm{CaO}(\%)$ & MgO (\%) & $\begin{array}{l}\text { OUTRAS IMPU- } \\
\text { REZAS }\end{array}$ \\
\hline Sulfato de cálcio P.A. & 0,0 & 0,0 & 0,0 & 46,4 & 32,5 & 0,0 & 0,0 \\
\hline Óxido de silício P.A. & 83,6 & 0,0 & 0,0 & 0,0 & 0,0 & 0,0 & 0,0 \\
\hline Óxido de Ferro P.A. & 0,0 & 0,0 & 96,5 & 0,0 & 0,0 & 0,0 & 0,0 \\
\hline Óxido de Alumínio P.A. & 0,0 & 96,4 & 0,0 & 0,0 & 0,0 & 0,0 & 0,0 \\
\hline Carbonato de cálcio P.A. & 0,0 & 0,0 & 0,0 & 0,0 & 56,0 & 0,0 & 0,0 \\
\hline Calcário & 17,6 & 3,1 & 1,8 & 0,3 & 59,0 & 5,4 & 1,3 \\
\hline Bauxita & 10,0 & 75,0 & 12,7 & - & 0,0 & 0,1 & 2,0 \\
\hline Lodo de anodização seco & 1,9 & 73,6 & 0,5 & 20,5 & 0,6 & 0,2 & 2,6 \\
\hline
\end{tabular}

A Tabela 3 apresenta as composições de fases dos clínqueres experimentais calculadas pelo método de BOGUE [20] adaptado, considerando o proporcionamento do clínquer referência, baseado em CHEN [10]. A Tabela 4 apresenta o percentual calculado em massa de cada matéria-prima na farinha do respectivo clínquer $\mathrm{CSAB}$

Tabela 3: Composição teórica de fases dos clínqueres CSAB experimentais.

\begin{tabular}{l|l|l|l}
\hline FASES & CSAB I- REFERÊNCIA (\%) & CSAB II - 50\% LODO (\%) & CSAB III - 100\% LODO (\%) \\
\hline Ferrita - $\mathrm{C}_{4} \mathrm{AF}$ & 10,0 & 8,9 & 8,9 \\
\hline Ye'elimita - $\mathrm{C}_{4} \mathrm{~A}_{3} \$$ & 40,0 & 37,8 & 37,8 \\
\hline Belita - $\mathrm{C}_{2} \mathrm{~S}$ & 40,0 & 39,0 & 39,0 \\
\hline Anidrita - C $\$$ & 10,0 & 8,8 & 8,4 \\
\hline Cal livre - C & 0,0 & 0,0 & 0,0 \\
\hline Periclásio - MgO & 0,0 & 3,8 & 4,0 \\
\hline Outras impurezas & 0,0 & 1,6 & 1,8 \\
\hline
\end{tabular}

Tabela 4: Proporcionamento das matérias-primas das farinhas precursoras.

\begin{tabular}{l|l|l|l}
\hline MATÉRIAS-PRIMAS & CSAB I - REFERÊNCIA (\%) & CSAB II - 50\% LODO (\%) & CSAB III - 100\% LODO (\%) \\
\hline Carbonato de cálcio P.A. & 51,1 & 1,1 & - \\
\hline Sulfato de cálcio P.A. & 17,5 & 11,9 & 7,3 \\
\hline Óxido de Alumínio P.A. & 16,8 & - & - \\
\hline Óxido de silício P.A. & 12,2 & - & 0,2 \\
\hline Óxido de Ferro P.A. & 2,5 & - & 1,1 \\
\hline Calcário alto & - & 68,6 & 72,9 \\
\hline Bauxita & - & 9,2 & - \\
\hline Lodo de anodização seco & & 9,2 & 18,4 \\
\hline
\end{tabular}

\subsection{Análises termogravimétricas}

A Figura 2 compara os termogramas (TG) das três farinhas precursoras. Observa-se nas curvas de TG que as farinhas compostas com resíduo apresentam maior perda de massa, isto se deve a decomposição do lodo. 


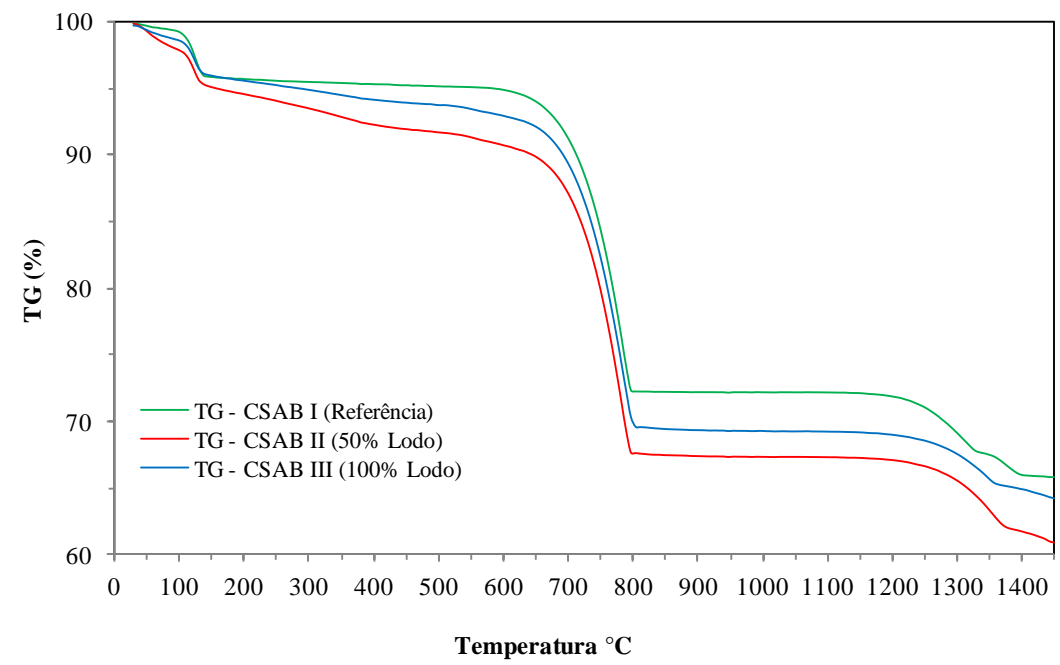

Figura 2: Curva TG das farinhas sob atmosfera de nitrogênio e taxa de aquecimento de $10^{\circ} \mathrm{C} / \mathrm{min}$.

Entre as temperaturas de 580 a $990^{\circ} \mathrm{C}$, verifica-se a maior porcentagem de perda de massa, decorrente da descarbonatação da calcita, associada a emissão química de $\mathrm{CO}_{2}$ para a atmosfera. Assim, as farinhas com resíduo apresentaram maior emissão química de $\mathrm{CO}_{2}$. Isto se deve a menor quantidade de gipsita nessas farinhas, a qual é uma fonte de $\mathrm{CaO}$ sem emissão de $\mathrm{CO}_{2}$. Como o lodo foi fonte de sulfato e este não era sulfato de cálcio, utilizou-se mais calcário para atender a demanda de $\mathrm{CaO}$ para formação das fases do clínquer. Considerando a perda de massa total média igual a $32 \%$ e a perda média por descarbonatação igual a $24 \%$, tem-se que seriam necessárias 1,47 ton de farinha para produção de 1,0 ton de clínquer. A emissão química de $\mathrm{CO}_{2}$ por tonelada do clínquer referência seria $336 \mathrm{~kg}$, e o aumento médio devido à utilização do lodo seria de $17 \%$. Esses resultados de emissão de $\mathrm{CO}_{2}$ estão coerentes com os obtidos por COSTA [17], que também calculou a emissão por tonelada de clínquer Portland em torno de $501 \mathrm{~kg}$. Após os $1200^{\circ} \mathrm{C}$, observa-se a decomposição dos compostos sulfatados.

A Tabela 5 apresenta o valor da perda de massa para cada farinha precursora nas temperaturas de $1250^{\circ} \mathrm{C}$ e $1450^{\circ} \mathrm{C}$. Verifica-se que diferença da perda de massa média é de $5 \%$ aproximadamente. Além de o consumo energético ser maior quando se utiliza a temperatura máxima de queima de $1450^{\circ} \mathrm{C}$, tem-se também perda do produto por decomposição das fases. A perda de massa de $1250^{\circ} \mathrm{C}$ a $1450^{\circ} \mathrm{C}$ indica redução do percentual de decomposição de compostos sulfatados, como o $\mathrm{C}_{4} \mathrm{~A}_{3} \$$, que é estável até cerca de $1350^{\circ} \mathrm{C}$. A Figura 3 compara as derivadas da curva de TG (DTG) das três farinhas CSAB.

Tabela 5: Perda de massa total das farinhas precursoras.

\begin{tabular}{l|l|l}
\hline FARINHA PRECURSORA & $\begin{array}{l}\text { ATÉ 1250ㄷ (TEMPERATURA DE } \\
\text { CLINQUERIZAÇÃO) }\end{array}$ & $\begin{array}{l}\text { ATÉ 1450C (TEMPERATURA DE EX- } \\
\text { TRAPOLAÇÃO) }\end{array}$ \\
\hline CSAB I - Referência & $28,90 \%$ & $34,16 \%$ \\
\hline CSAB II - 50\% Lodo & $33,30 \%$ & $39,04 \%$ \\
\hline CSAB III - 100\% Lodo & $31,45 \%$ & $35,18 \%$ \\
\hline
\end{tabular}




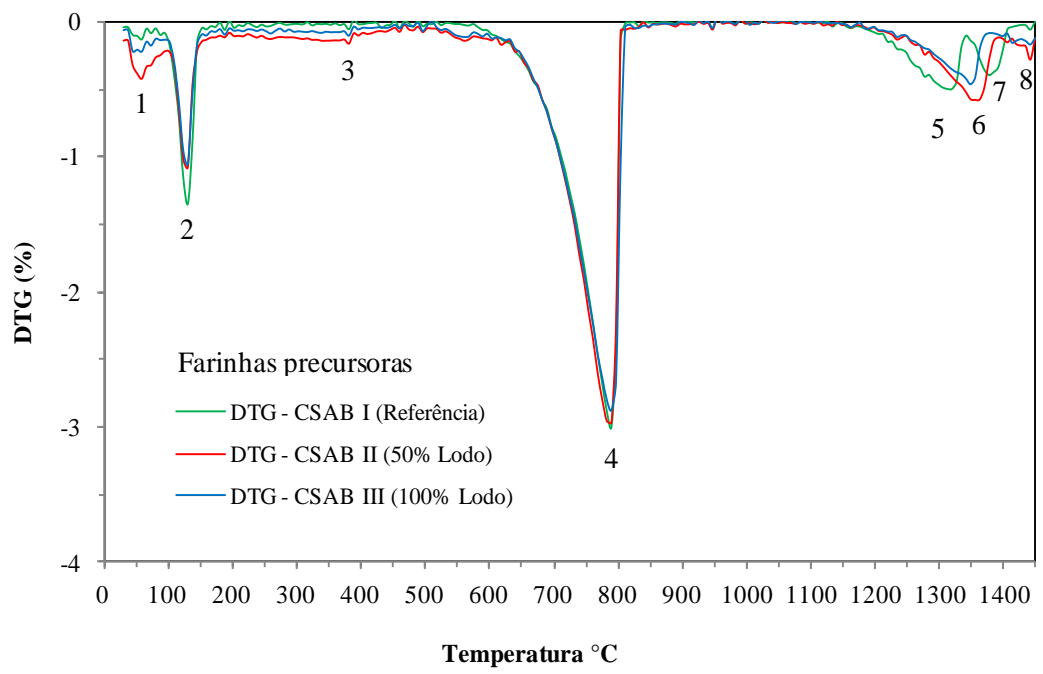

Figura 3: Curvas DTG das farinhas sob atmosfera de nitrogênio e taxa de aquecimento de $10^{\circ} \mathrm{C} / \mathrm{min}$.

A Tabela 6 apresenta a tradução dos picos endotérmicos identificados na Figura 3. A avaliação da perda de massa da farinha $\mathrm{CSAB}$ até a temperatura de $1450^{\circ} \mathrm{C}$ mostrou que existe uma emissão de $\mathrm{SO}_{\mathrm{x}}$ pela decomposição de compostos sulfatados, como as fases ye'elimita e anidrita. Vale ressaltar que a anidrita decompõe-se a partir da temperatura de $1200^{\circ} \mathrm{C}$ [27]. Tem-se também que entre 1000 e $1250^{\circ} \mathrm{C}$ ocorre a formação da ye'elimita e acima dessa temperatura o $\mathrm{C}_{4} \mathrm{~A}_{3} \overline{\mathrm{S}}$ se decompõe: reações com a cal livre, caso presente, para formar $\mathrm{C}_{3} \mathrm{~A}_{\text {e }} \mathrm{CaSO}_{4}$ [16]. A emissão de $\mathrm{SO}_{x}$ pode ser controlada através da temperatura de sintetização e decomposição das fases do clínquer CSAB. Portanto, a temperatura de $1250^{\circ} \mathrm{C}$ pode garantir uma queima com reduzida emissão de $\mathrm{SO}_{\mathrm{x}}$. Foi observado também que farinhas compostas com lodo de anodização do alumínio possuem perda de massa próximas a da farinha referência, este fato demonstra que o teor de sulfato do lodo contribui para formação das fases, reduzindo o consumo de gipsita. Por fim, o aproveitamento de resíduos para a produção do cimento $\mathrm{CSAB}$ é um caminho para viabilização deste material de reduzida emissão química de $\mathrm{CO}_{2}$, porém a temperatura de queima deve ser controlada para se evitar a emissão da mesma forma de $\mathrm{SO}_{\mathrm{x}}$, o que pode implicar danos ambientais e transtornos no processo industrial, além de evitar a decomposição de fases sintéticas importantes para o desempenho do material quando aplicado em obras.

Tabela 6: Eventos determinados na TG/DTG para as farinhas precursoras dos clínqueres CSAB. 


\begin{tabular}{|c|c|c|c|c|c|}
\hline EVENTO & $\begin{array}{l}\text { FARINHA } \\
\text { CSAB }\end{array}$ & FENÔMENO & $\begin{array}{l}\text { TEMPERATURA } \\
\text { DE PICO (ㅇ) }\end{array}$ & ${ }^{*} \mathrm{TP} .\left({ }^{\circ} \mathrm{C}\right)$ & $\begin{array}{l}\text { PERDA DE } \\
\text { MASSA (\%) }\end{array}$ \\
\hline \multirow{3}{*}{1} & I & \multirow{3}{*}{ Perda de umidade } & \multirow{3}{*}{$30-100[28]$} & \multirow{3}{*}{50} & 0,83 \\
\hline & II & & & & 2,15 \\
\hline & III & & & & 1,34 \\
\hline \multirow{3}{*}{2} & I & \multirow{3}{*}{$\begin{array}{l}\text { Desidratação: } \\
\text { Gipsita - Ca- } \\
\mathrm{SO}_{4} \cdot 2 \mathrm{H}_{2} \mathrm{O}\end{array}$} & \multirow{3}{*}{$100-260[28]$} & \multirow{3}{*}{130} & 3,66 \\
\hline & II & & & & 3,88 \\
\hline & III & & & & 3,41 \\
\hline \multirow{3}{*}{3} & I & \multirow{3}{*}{$\begin{array}{l}\text { Desidratação: } \\
\mathrm{Al}(\mathrm{OH})_{3} ; \\
\mathrm{FeO} . \mathrm{OH} ; \\
\mathrm{MgCO}_{3} .3 \mathrm{H}_{2} \mathrm{O} \text { e } \\
\text { outras fases não } \\
\text { identificadas }\end{array}$} & \multirow{3}{*}{$260-580[28]$} & \multirow{3}{*}{375} & 0,69 \\
\hline & II & & & & 3,63 \\
\hline & III & & & & 2,42 \\
\hline \multirow{3}{*}{4} & I & \multirow{3}{*}{$\begin{array}{l}\text { Decomposição: } \\
\text { Carbonato de cál- } \\
\text { cio }-\mathrm{CaCO}_{3}\end{array}$} & \multirow{3}{*}{$580-990[28]$} & \multirow{3}{*}{790} & 22,83 \\
\hline & II & & & & 23,83 \\
\hline & III & & & & 24,11 \\
\hline $5 ; 7$ e 8 & I & \multirow{3}{*}{$\begin{array}{l}\text { Decomposição } \\
\text { dos compostos } \\
\text { sulfatados: } \mathrm{C}_{4} \mathrm{~A}_{3} \$ ; \\
\mathrm{CaSO}_{4}\end{array}$} & \multirow{3}{*}{$\begin{array}{l}1200-1450 \\
{[16,27]}\end{array}$} & $1300 ; 1370 ; 1440$ & 6,29 \\
\hline 6 e 8 & II & & & $1350 ; 1440$ & 6,34 \\
\hline 6 e 8 & III & & & $1350 ; 1440$ & 4,96 \\
\hline
\end{tabular}

* temperatura no pico na curva DTG.

Segundo SOUZA [25] o $\mathrm{SO}_{2}$ (dióxido de enxofre) é o mais comum poluente formado pela utilização de combustíveis nas cimenteiras para queimar a matéria-prima e gerar o clínquer. Conforme o autor, o volume de combustível consumido pela indústria do cimento gera vários outros poluentes além do $\mathrm{SO}_{2}$, tais como: dióxido de carbono $\left(\mathrm{CO}_{2}\right)$, óxidos de nitrogênio $\left(\mathrm{NO}_{\mathrm{x}}\right)$ e, óxidos de enxofre $\left(\mathrm{SO}_{\mathrm{x}}\right) \cdot \mathrm{O} \mathrm{NO}_{\mathrm{x}}$ resultante em uma indústria cimenteira pode ser: $\mathrm{NO}_{\mathrm{x}}$ térmico, produto da oxidação do nitrogênio atmosférico em temperaturas elevadas, e o $\mathrm{NO}_{\mathrm{x}}$ do combustível, formado pela oxidação do nitrogênio no combustível [26]. Para o autor, quando se diminui a temperatura de chama ocorre a redução do $\mathrm{NO}_{\mathrm{x}}$ térmico, já que a temperatura é menor. Assim, clínqueres produzidos em temperaturas mais baixas de queima podem contribuir para redução do impacto negativo ambiental gerado pela produção do cimento.

Foi observada, nos resultados da análise experimental desta pesquisa, uma perda de massa de 6,29\% da farinha referência entre as temperaturas de 1200 a $1450^{\circ} \mathrm{C}$. Considerando que tenha ocorrido a decomposição da fase $\mathrm{C}_{4} \mathrm{~A}_{3} \$$, seriam emitidos $25,16 \mathrm{~kg}$ de $\mathrm{SO}_{2} /$ ton de clínquer CSAB produzido, e $18,75 \mathrm{~kg} \mathrm{de} \mathrm{SO}_{2} /$ ton de clínquer no caso da substituição total da bauxita pelo lodo de anodização do alumínio.

A prática do coprocessamento de resíduos em fornos de cimento ainda demanda muitos estudos, visando-se elucidar os aspectos da real contribuição do coprocessamento de resíduos e o estabelecimento dos limites e riscos a ele associados, em processos onde um rígido controle das condições operacionais e um monitoramento contínuo e eficaz do processo e das características físicas e químicas dos resíduos não forem praticados [1]. Concordando com a autora, salienta-se que não apenas a decomposição de fases sulfatadas deve ser investigada, mas sim de todos os sólidos que podem volatilizar ao longo da temperatura de queima, principalmente quando houver combinações de matérias-primas/resíduos/subprodutos que possam gerar gases poluente e irritantes.

\section{CONCLUSÕES}

As farinhas CSAB proporcionadas com lodo de anodização do alumínio possuem perda de massa total superior quando comparadas à referência, isto se deve a desidratação do hidróxido de alumínio presente no resí- 
duo, em sua maior parcela. A perda de massa pela decomposição de fases sulfatadas é inferior quando existe a substituição total da bauxita pelo resíduo na composição da farinha CSAB. A maior incorporação de enxofre nos cristais da fase belita pode ter contribuído para o fato. Foi observada diferença da perda de $\mathrm{SO}_{2}$ de aproximadamente $26 \%$ entre farinha dosadas com reagentes químicos puros e farinhas com substituição total da bauxita por lodo de anodização do alumínio, considerando que tenha ocorrido a decomposição da fase $\mathrm{C}_{4} \mathrm{~A}_{3} \$$ entre 1200 a $1450^{\circ} \mathrm{C}$.

\section{AGRADECIMENTOS}

Os autores agradem às instituições que possibilitaram a realização desta pesquisa. Assim, profundos agradecimentos ao Núcleo Orientado para a Inovação da Edificação (NORIE) da Universidade Federal do Rio grande do Sul (UFRGS, Brasil) e ao Laboratório de Materiais Cerâmicos (LACER/UFRGS). Um agradecimento especial a CAPES (Brasil) pelo auxílio financeiro ao autor principal e ao CNPq (Edital Universal e Produtividade em Pesquisa) pelo apoio financeiro para realização da parte experimental desta proposta.

\section{BIBLIOGRAFIA}

[1] ROCHA, S. D. F., LINS, V. F. C., ESPIRITO SANTO, B. C., "Revisão da Literatura: aspectos do coprocessamento de resíduos em fornos de clínquer”, Rev. Eng. Sanit. Ambient., v.16, n.1, pp. 1-10, jan/mar 2011.

[2] MEHTA, P. K, MONTEIRO, P. J. M. Concreto: Microestrutura, Propriedades e Materiais. 2008. São Paulo: IBRACON, $1^{\text {a }}$ Ed., p. 289-307.

[3] POTGIETER, H. J. "An Overview of Cement production: How ."green.” And sustainable is the industry?”Environmental Management and Sustainable Development. v. 1, n. 2, 2012.

[4] KOHLHAAS, A., LABAN, A.O. (Cement Handbook (2nd Edn). Bauverlag GmbH. Wiesbaden und Berlin, Germany, 1983.

[5] USGS. U.S. Geological Survey Minerals Yearbook. Volume I - Metals and Minerals. Cement Statistics and Information. 2013. Disponível em: http://minerals.er.usgs.gov/minerals/pubs/commodity/cement/mcs2013-cemen.pdf. Acesso em: 08 de julho de 2013.

[6] EIA. International Energy Annual 2004. US Energy Information Administration. 2006. Disponível em: http://www.eia.doe.gov/iea/.

[7] BRASIL. Balanço Energético Nacional (2005). Ministério de Minas e Energia. Brasília. 2005. Disponível em: http://www.mme.gov.br. Acesso em: 1 set. 2011.

[8] EPA. Emission Facts: Average Carbon Dioxide Emissions Resulting from Gasoline and Diesel Fuel. U.S. Environmental Protection Agency. 2005. Disponível em: http://www.epa.gov/otaq/climate/420f05001.htm. [9] OLIVEIRA, M. E. D., VAUGHAN, B.E., et al., "Ethanol as fuel: energy, carbon dioxide balances and ecological footprints", BioScience, v.55, n.7, pp.593-602, 2005.

[10] CHEN, I. A. Synthesis of Portland Cement and Calcium Sulfoaluminate-Belite Cement for Sustainable Development and Performance. PhD dissertation. Department of Civil Engineering of The University of Texas at Austin, 2009. 174 p.

[11] JEWELL, R. B., RATHBONE, R. F., ROBL, T. L., et al., Fabrication and Testing of CSAB Cements in Mortar and Concrete that Utilize Circulating Fluidized Bed Combustion Byproducts. 2009 World of Coal Ash (WOCA) Conference - May 4-7, 2009 in Lexing, KY, USA. Disponível em: http://www.flyash.info/2009/108-jewell2009.pdf. Acesso em: 12/10/2012.

[12] MARTÍN-SEDEÑO, M. C., CUBEROS, A. J. M., DE LA TORRE, Á. G., "Aluminum-rich belite sulfoaluminate cements: Clinkering and early age hydration", Cement and Concrete Research, v.40, n.3, pp. 359-369.

[13] SENFF, A., CASTELA, W., HAJJAJI, D., et al., "Formulations of sulfobelite cement through design of experiments", Construction and Building Materials,v.25, n. 8, Aug. 2011, pp. 3410-3416.

[14] ÁlvarEZ-PINAZO, A., CUESTA, M., GARCÍA-MATÉ, I., et al., “Aranda, Rietveld quantitative phase analysis of Yeelimite-containing cements", Cement and Concrete Research, v. 42, n. 7, July 2012, pp. 960-971.

[15] TAYLOR, H.F.W. Cement Chemestry, British Library, 1992.

[16] ODLER, I. Special inorganic cements, Modern Concrete Technology, E \& FN SPON Editor, 2000.

[17] COSTA, E.B. Aproveitamento do resíduo de anodização do alumínio na produção do cimento sulfoalu- 
minato de cálcio belítico, Dissertação (Mestrado em Construção Civil), Programa de Pós-Graduação em Engenharia Civil, UFRGS, Porto Alegre, 2013.

[18] ANDAC, M., GLASSER, F.P. Microstructure and microchemistry of calcium sulfoaluminate, Materiais Research Society Proccedings , v. 370, pp.135-142, 1995.

[19] CENTURIONE, S. L. Influência das características das matérias-primas no processo de sinterização do clínquer Portland. (Dissertação de Mestrado). Instituto de Geociências, Universidade de São Paulo (USP), São Paulo, 1993. 154 p.

[20] BOGUE, R. H., "Calculation of the Compounds in Portland Cement". Analytical Edition, Industrial and Engineering Chemistry, v. 1, n. 4, pp. 192-197. October 15, 1929.

[21] MAJLING, J., ROY, D.M. The potential of flyash for cement manufacture, Am. Ceram. Soc. Bull, v. 72 n.10, pp.77-80, 1993.

[22] PEREIRA, F.R. Valorização de resíduos industriais como fonte alternativa mineral: composições cerâmicas e cimentíceas. Portugal, 2006. Tese de doutoramento. Aveiro, Universidade de Aveiro, Portugal.

[23] MARINGOLO, V. Clínquer co-processado: produto de tecnologia integrada para a sustentabilidade e competitividade da indústria de cimento. São Paulo, 2001. Tese de Doutorado. Mineralogia Aplicada. Departamento de Mineralogia e Petrologia, São Paulo: Universidade de São Paulo.

[24] CHOTOLI, F. F. Obtenção de clínquer de cimento a partir de escória de aciaria a oxigênio: estudo em escala laboratorial. Instituto de Pesquisas Tecnológicas (IPT), São Paulo, 2006.

[25] SOUZA, A. C. "Otimização Global Estocástica Multi-Objetivos na Produção de Cimento com CoProcessameno de Resíduos e Adição de Mineralizadores", Exame de Qualificação, Universidade Federal de Itajubá, UNIFEI, Itajubá-MG, 2007.

[26] TOSTA. L. I., SOUZA, A. C., ROGÉRIO, J. S. Gestão da energia na produção de cimento portland com uso de mineralizadores e combustíveis alternativos. In: Encontro Nacional de Engenharia de Produção, XXVII, pp. 1-10, 2007.

[27] FILIPPIDIS, A., GEORGAKOPOULOS, A., KASSOLI-FOURNARAKI, A. Mineralogical components of some thermally decomposed lignite and lignite ash from the Ptolemais basin, Greece, International Journal of Coal geology, v.30, pp.303-314, 1996.

[28] MARTINS, F. M. Caracterização química e mineralógica de resíduos sólidos industriais minerais do estado do Paraná, Dissertação de Mestrado, Curso de Pós graduação em Química, Universidade do Paraná, Curitiba, 2006. 